\title{
4 Nahrungsergänzungsmittel
}

\author{
Stephanie Thomas, Sonja Grunewald und Uwe Paasch
}

\begin{abstract}
Die männliche Infertilität ist häufig multifaktorieller Genese und es besteht ein großer Drang seitens der Betroffenen, durch aktive Beteiligung die Situation zu verbessern. Interessante Angriffspunkte sind dabei sogenannte Lifestyle-Faktoren wie Sport, Rauchen und Drogen. Gern wird in der öffentlichen Wahrnehmung unterstellt, dass die Ernährung am leichtesten zu beeinflussen sei. Dieses Kapitel gibt Aufschluss über die Sinnhaftigkeit der Einnahme von Nahrungsergänzungsmitteln. Der Fokus wird auf solche Stoffe gelegt, zu denen ausreichend Informationen verfügbar sind: Zink, Carnitin, Folsäure, Selen, Vitamine, Coenzym Q und langkettige Fettsäuren. Ergänzend wurden Informationen zum Inhalt häufig eingesetzter Nahrungsergänzungsmittel (NEM), soweit verfügbar, zusammengetragen (s. Tab. 1).
\end{abstract}

\subsection{Zink}

Zink ist ein essenzielles Spurenelement für den Menschen und spielt wie bei allen schnell differenzierenden Epithelien auch in der Reproduktion eine entscheidende Rolle. Dies unterstreichen schon frühe Human- und Tierstudien, bei denen ein Zinkdefizit zur Infertilität führte (Barney et al. 1968; Sandstead et al. 1967).

Zink kann verschiedene Spermienparameter beeinflussen. Während einige Studien eine signifikant positive Korrelation zwischen der Zinkkonzentration im Seminalplasma und der Spermienzahl bzw. Motilität zeigen (Chia et al. 20oo; Colagar et al. 2009; Liu et al. 2009; Wong et al. 2001; Xu et al. 1993), findet sich dieser Zusammenhang (Liu et al. 2009; Saaranen et al. 1987) bzw. eine Korrelation zur Spermienmorphologie in anderen Studien nicht (Fuse et al. 1999; Liu et al. 2009; Saaranen et al. 1987). Weiterhin ist umstritten, ob sich die Zinkkonzentration im Seminalplasma von fertilen im Vergleich zu infertilen Männern unterscheidet (Chia et al. 200o; Colagar et al. 2009) oder nicht (Fuse et al. 1999; Wong et al. 2001).

Ein möglicher Grund für unterschiedliche Zinkkonzentrationen bei fertilen und infertilen Männern könnte die ansteigende Zahl der abnormen Spermien bei infertilen Männern sein (Menkveld et al. 199o). Diese geht mit einer Zunahme reaktiver Sauer- 
Tab. 1 Vergleich von NEM zur Behandlung der männlichen Sub- bzw. Infertilität

\begin{tabular}{|c|c|c|c|c|c|}
\hline & \multicolumn{2}{|c|}{$\begin{array}{l}\text { Orthomol Fertil plus } \\
\text { Tabletten + Kapsel }\end{array}$} & \multicolumn{2}{|c|}{ Profertil Kapseln } & \multirow{2}{*}{$\begin{array}{l}\text { Menevit } \\
\text { Kapseln } \\
1 \text { Kapsel }\end{array}$} \\
\hline & pro Tag & pro $100 \mathrm{~g}$ & pro Tag & pro $100 \mathrm{~g}$ & \\
\hline \multicolumn{6}{|l|}{ Vitamine } \\
\hline natürliches Vitamin E (TE) & $120 \mathrm{mg}$ & $4,1 \mathrm{~g}$ & $120 \mathrm{mg}$ & $7,0 \mathrm{~g}$ & $400 \mathrm{IU}$ \\
\hline Vitamin C & $250 \mathrm{mg}$ & $8,6 \mathrm{~g}$ & - & - & $100 \mathrm{mg}$ \\
\hline Vitamin B6 & $3,5 \mathrm{mg}$ & $121 \mathrm{mg}$ & - & - & - \\
\hline Vitamin B12 & $9 \mu g$ & $310 \mu g$ & - & - & - \\
\hline Folsäure & $800 \mu g$ & $28 \mathrm{mg}$ & $800 \mu g$ & $46,5 \mathrm{mg}$ & $500 \mu g$ \\
\hline \multicolumn{6}{|l|}{ Spurenelemente } \\
\hline Zink & $40 \mathrm{mg}$ & $1,4 \mathrm{~g}$ & $40 \mathrm{mg}$ & $2,3 \mathrm{~g}$ & $25 \mathrm{mg}$ \\
\hline Selen & $80 \mu g$ & $2,8 \mathrm{mg}$ & $60 \mu \mathrm{g}$ & $3,5 \mathrm{mg}$ & $26 \mu g$ \\
\hline Kupfer & $1 \mathrm{mg}$ & $34 \mathrm{mg}$ & - & - & - \\
\hline \multicolumn{6}{|l|}{ weitere Mikronährstoffe } \\
\hline L-Carnitin & $440 \mu g$ & $15 \mathrm{~g}$ & $440 \mathrm{mg}$ & $25,6 \mathrm{~g}$ & - \\
\hline N-Acetylcystein & $80 \mathrm{mg}$ & $2,8 \mathrm{~g}$ & - & - & - \\
\hline L-Arginin & - & - & $250 \mathrm{mg}$ & $14,5 \mathrm{~g}$ & - \\
\hline Glutathion & - & - & $80 \mathrm{mg}$ & $4,7 \mathrm{~g}$ & - \\
\hline Coenzym Q10 & $15 \mathrm{mg}$ & $517 \mathrm{mg}$ & $15 \mathrm{mg}$ & $0,9 \mathrm{~g}$ & - \\
\hline $\begin{array}{l}\text { gemischte Carotinoide } \\
\text { enthalten } \beta \text {-Carotin } \\
\text { Lutein } \\
\text {, Lycopin }\end{array}$ & $3 \mathrm{mg}^{1,2,3}$ & $103 \mathrm{mg}^{1,2,3}$ & - & - & $6 \mathrm{mg}^{3}$ \\
\hline Omega-3-Fettsäuren, davon & $170 \mathrm{mg}$ & $5,9 \mathrm{~g}$ & - & - & - \\
\hline Eicosapentaensäure (EPA) & $90 \mathrm{mg}$ & $3,1 \mathrm{~g}$ & - & - & - \\
\hline Docosahexaensäure (DHA) & $65 \mathrm{mg}$ & $2,2 \mathrm{~g}$ & - & & - \\
\hline physiologischer Brennwert & $22 \mathrm{~kJ}(5,1 \mathrm{kcal})$ & $720 \mathrm{~kJ}$ (171 kcal) & & - & - \\
\hline Eiweiß & $0,2 \mathrm{~g}$ & $5,5 \mathrm{~g}$ & - & - & - \\
\hline Kohlenhydrate & $0,4 \mathrm{~g}$ & $14 \mathrm{~g}$ & - & - & - \\
\hline Fett & $0,4 \mathrm{~g}$ & $13 \mathrm{~g}$ & - & - & - \\
\hline Broteinheiten (BE) & $<0,1 \mathrm{BE}$ & $1 \mathrm{BE}$ & - & - & - \\
\hline Knoblauchöl & - & - & - & - & $333 \mu g$ \\
\hline Packungsgröße & 90 Stück & 180 Stück & & & 90 Stück \\
\hline Einnahme pro Tag & 3 Tabl. + 1 Kapsel & 2 Kapseln & & & 1 Kapsel \\
\hline
\end{tabular}

stoffspezies (ROS) einher (Rao et al. 1989), wodurch die antioxidative Kapazität und auch die Zinkspiegel im Seminalplasma sinken. Zink besitzt eine antioxidative Wirkung (Gavella u. Lipovac 1998; Omu et al. 2008). Es fungiert als Scavenger für Super- 
oxidanionen (Gavella u. Lipovac 1998) und stabilisiert das Chromatin im Spermiumnukleus (Kvist et al. 1990). So lassen sich Effekte einer oralen Zinktherapie bei infertilen Männern wie ein Anstieg der Spermienzahl (Omu et al. 1998; Tikkiwal et al. 1987) und die Verbesserung der Motilität (Kynaston et al. 1988; Omu et al. 1998; Tikkiwal et al. 1987) erklären.

Zusammenfassend lässt sich derzeit schlussfolgern, dass sich bei Patienten mit Asthenozoospermie möglicherweise ein Nutzen der oralen Zinktherapie abzeichnet.

\subsection{Carnitin}

Carnitin ist vor allem als Nahrungsergänzungsmittel für Sportler und Übergewichtige bekannt, da es neben seiner antioxidativen Wirkung (Vicari u. Calogero 2001) die Fettsäureoxidation unterstützen soll. Bei der mitochondrialen $\beta$-Oxidation spielt Carnitin eine essenzielle Rolle. In der inneren Mitochondrienmembran dient es als Shuttle für langkettige Acyl-Coenzym A-Moleküle (Siliprandi et al. 1989). Es wird zu 75\% aus der Nahrung und zu 25\% in den Hepatozyten aus Lysin und Methionin synthetisiert. Als freies L-Carnitin wird es aus dem Blutplasma über membranständige $\mathrm{Na}^{+}-$ abhängige Carnitintransporter OCTN1-3 in die Epididymisflüssigkeit und in die Spermatozoen transportiert und akkumuliert als freies und acetyliertes L-Carnitin (Xuan et al. 2003). Mit Beginn der Spermienmotilität im epididymalen Lumen steigt die LCarnitin Konzentration an, während des Transits der Spermatozoen vom Kaput zur Kauda akkumuliert freies L-Carnitin intrazellulär und wird von den Spermatozoen sofort acetyliert (Jeulin et al. 1987). Dies impliziert, dass die immaturen Spermien während ihrer Reifung durch Carnitin ihre Motilität erlangen (Hinton et al. 1981; Jeulin et al. 1987). Diese Erkenntnisse führten zu der Hypothese, Carnitin könnte die Spermienmotilität bei infertilen Patienten mit Oligozoospermie bzw. Asthenozoospermie steigern. Mehrere, z.T. Placebo-kontrollierte Studien zeigten eine Verbesserung der Gesamt- und Progressivmotilität unter Einnahme von Carnitin bzw. L-Acetyl-Carnitin (Cavallini et al. 2004; Costa et al. 1994; Lenzi et al. 2003; Lenzi et al. 2004; Vicari u. Calogero 2001). Uneinheitlich ist die Datenlage im Hinblick auf die Spermienzahl und -konzentration. Es wurde einerseits ein signifikanter Anstieg der Spermienzahl gemessen (Cavallini et al. 2004; Costa et al. 1994; Lenzi et al. 2003), aber nicht immer verifiziert (Lenzi et al. 2004). Hinsichtlich der Wirkung von Carnitin auf die Spermienmorphologie gehen die Meinungen in der Literatur ebenfalls auseinander (Cavallini et al. 2004; Khademi et al. 2005; Lenzi et al. 2003; Lenzi et al. 2004). Dabei wurden meist 2-4 g Carnitin/Tag an die Probanden verabreicht, mitunter auch Acetylcarnitin in Kombination mit Carnitin (Lenzi et al. 2004). Das lässt vermuten, dass die Carnitinspiegel im Seminalplasma bei Intervention ansteigen, jedoch konnte dies nicht sicher nachvollzogen werden (Lenzi et al. 2003; Lenzi et al. 2004).

Obwohl erste Analysen infertiler und fertiler Probanden keine Unterschiede der Carnitinkonzentration im Seminalplasma zeigten (Kohengkul et al. 1977), deuten neuere Studien darauf hin, dass die Carnitinspiegel im Seminalplasma bei infertilen Patienten signifikant geringer sind als bei fertilen (Sheikh et al. 2007; Zopfgen et al. 2000).

Aus den vorhandenen Studien lässt sich schließen, dass Carnitin infolge seiner antioxidativen Potenz eine wirksame alternative Therapieoption darstellen könnte, 
wenn erhöhte ROS-Spiegel ursächlich für beeinträchtigte Spermatozoenfunktion sein können.

\subsection{Folsäure}

Das Vitamin Folsäure ist für die DNA-, RNA- und Proteinsynthese und somit auch während der Spermatogenese essenziell. Der tägliche Bedarf dieses B-Vitamins beträgt $400 \mu \mathrm{g}$ beim sonst gesunden Menschen, die Aufnahme über die Nahrung ist jedoch marginal (De Bree et al. 1997). Bei einer Folsäuresupplementierung gilt es zu beachten, dass die hämatologischen Befunde eines Vitamin $\mathrm{B}_{12}$-Mangels überdeckt werden könnten. Aus diesem Grund ist es wichtig, vor einer Folsäureintervention die Cobalaminspiegel zu bestimmen.

Die orale Folsäuregabe steigert signifikant den Folsäurespiegel im Seminalplasma (De Bree et al. 1997; Landau et al. 1978; Wong et al. 2002). Jedoch hat die alleinige Verabreichung von Folsäure keinen Einfluss auf Spermienkonzentration, Motilität und Morphologie (Landau et al. 1978; Wong et al. 2002). Auch die kombinierte Zink/Folsäure-Therapie erbrachte nur bei subfertilen Männern einen signifikanten Anstieg der Spermienzahl - unter Vergrößerung des Anteils pathomorpher Spermien. Bei fertilen Probanden zeigte sich keine Veränderung der Spermiogrammparameter (Wong et al. 2002).

Ursächlich für einen ausbleibenden Effekt könnte mitunter die kurze Verabreichungsdauer von 30 Tagen sein. Eine Veränderung der Spermienzahlen würde man voraussichtlich nach einem Durchlauf der Spermatogenese, die ca. 74 Tage andauert, sehen. Bei der Messung von Folat kann außerdem zwischen 5-Methyltetrahydrofolat, der Folathauptform im Blutplasma und den Nicht-Methyltetrahydrofolaten (Tetrahydrofolat, 10-Formyltetrahydrofolat, 5,10-Methylentetrahydrofolat) unterschieden werden. In diesem Fall zeigte sich eine signifikant positive Korrelation zwischen der Konzentration an Nicht-Methyltetrahydrofolaten im Seminalplasma und der Spermiendichte bzw. totaler Spermienzahl (Wallock et al. 2001). Auch durch Gabe von 5-Formyltetrahydrofolat für 3 Monate zeigte sich eine Verbesserung der Spermienzahl und Motilität (Bentivoglio et al. 1993).

Neuere Studien berücksichtigen zusätzlich Effekte der Folsäuresupplementation auf die DNA-Integrität. Bei fertilen Probanden korrelierte die Folatkonzentration im Seminalplasma invers mit dem DNA-Fragmentationsindex (DFI). Eine geringe Folatkonzentration im Seminalplasma war demzufolge mit einer erhöhten Zahl von DNAStrangbrüchen assoziiert (Boxmeer et al. 2009).

\subsection{Selen}

Selen ist Bestandteil verschiedener, sowohl in Spermien als auch im Seminalplasma vorhandener antioxidativ wirkender Enzyme (Selenoproteine), wie z.B. der Glutathionperoxidase (GPx) (Flohe et al. 1973; Rotruck et al. 1973) und der Phospholipidhydroperoxidglutathionperoxidase (GPx4) (Maiorino et al. 1989; Thomas et al. 1990).

Die GPx verhindert die Lipidperoxidation in der Spermienplasmamembran (Alvarez u. Storey 1989; Brown et al. 1977). Für die GPx4 konnte eine verringerte Aktivität bei infertilen Männern gezeigt werden (Foresta et al. 2002). 
Hinsichtlich der Korrelation des Selengehaltes im Seminalplasma zu den klassischen Spermiogrammparametern Spermienzahl, Motilität und Morphologie finden sich uneinheitliche Studienergebnisse. Es konnten sowohl positive (Bleau et al. 1984; Noack-Fuller et al. 1993; Oldereid et al. 1998; Xu et al. 1993; Xu et al. 2003), negative (Akinloye et al. 2005) als auch fehlende Zusammenhänge hergestellt werden (Behne et al. 1988; Roy et al. 1990; Saaranen et al. 1987; Xu et al. 1994).

Ein Vergleich der Selenkonzentration in Seminalplasmen fertiler und infertiler Männer erbrachte zunächst keine signifikanten Unterschiede (Saaranen et al. 1989), später konnten jedoch erniedrigte Selen-Level bei infertilen Männern nachgewiesen werden (Iwanier u. Zachara 1995). Auch Untersuchungen zur Selengabe zeigten inkonsistente Ergebnisse. Obwohl die Selen-Supplementierung subfertiler Männer zu einem Anstieg der Selenkonzentration im Seminalplasma führte (Iwanier u. Zachara 1995), konnte nur eine Studie einen signifikant positiven Einfluss auf die Motilität nachweisen (Scott et al. 1998). Alle anderen Untersuchungen fanden keinen Effekt auf die Spermienmotilität (Iwanier u. Zachara 1995), die Spermienkonzentration (Scott et al. 1998) und die Morphologie (Iwanier u. Zachara 1995).

Insgesamt lassen die konträren Studienergebnisse nicht sicher auf einen Nutzen einer Selengabe bei subfertilen/infertilen Männern schließen. Es fehlen Placebo-kontrollierte Studien mit ausreichend hohen Patientenzahlen, die ggf. auch Subgruppen der Patienten untersuchen.

\subsection{Vitamin C}

Vitamin C (Ascorbinsäure) gilt in der öffentlichen Wahrnehmung als wichtige antioxidativ wirksame und breit präventiv eingesetzte Substanz. Vitamin C ist als Scavenger von reaktiven Sauerstoffspezies tatsächlich in der Lage, humane Spermatozoen vor oxidativen Schäden zu schützen (Fraga et al. 1991).

Die Seminalplasma-Spiegel der Ascorbinsäure sind bei fertilen Männern signifikant höher als bei infertilen (Colagar u. Marzony 2009; Lewis et al. 1997; Mostafa et al. 2006; Thiele et al. 1995). Gleichzeitig findet sich eine positive Korrelation zwischen der Vitamin C-Konzentration im Seminalplasma und der Anzahl normomorpher Spermien (Colagar u. Marzony 2009; Mostafa et al. 2006; Thiele et al. 1995), der Spermienkonzentration (Mostafa et al. 2006) und der Motilität (Kao et al. 2008; Mostafa et al. 2006). Auch wenn diese positiven Mechanismen nicht immer nachvollzogen werden konnten (Song et al. 2006), findet sich unter Supplementierung von Ascorbinsäure ein signifikanter Anstieg der Spermienmotilität (Dawson et al. 1987; Piomboni et al. 2008) und eine signifikante Verringerung pathomorpher Spermatozoen (Dawson et al. 1987; Piomboni et al. 2008). Allerdings blieb ein signifikanter Anstieg der Spermienkonzentration aus (Dawson et al. 1987).

Raucher, die im Vergleich zu Nichtrauchern vermehrt oxidativem Stress ausgesetzt sind, zeigen nicht nur eine verminderte Vitamin C-Konzentration im Seminalplasma (Mostafa et al. 2006), sondern auch eine verminderte Spermienmotilität (Shaarawy u. Mahmoud 1982).

Neue Studien zur DNA-Integrität ergaben eine inverse Beziehung der Konzentration von Vitamin C im Seminalplasma zur Menge der oxidativ geschädigten SpermienDNA bei Konsum einer mit Vitamin C angereicherten Diät (Fraga et al. 1991). Eine 
geringe ( $<5 \mathrm{mg} / \mathrm{dl})$ Vitamin C-Konzentration im Seminalplasma ist mit vermehrten Schäden an der Spermien-DNA vergesellschaftet (Song et al. 2006) und kann damit direkt die Fertilität beeinträchtigen.

Für das Vitamin C ergeben sich damit erste aber schwache Anhaltspunkte, dass ein Mangel möglicherweise die Spermatozoenfunktion beeinträchtigen kann. Derzeit ist es jedoch schwierig tatsächliche Mangelzustände gezielt zu identifizieren und einer rationalen Substitution zuzuführen.

\subsection{Vitamin $\mathrm{E}$}

Das ebenfalls antioxidativ wirksame Vitamin $\mathrm{E}$ ( $\alpha$-Tocopherol) ist sowohl in humanen Spermien als auch im Seminalplasma nachweisbar, jedoch korrespondieren die jeweiligen Konzentrationen nicht miteinander. Innerhalb der Spermien fand sich eine signifikant positive Korrelation zwischen dem zellulären Vitamin E-Spiegel und den Enzymaktivitäten der Superoxidanionendismutase (SOD) und der GPx (Therond et al. 1996). Passend dazu sind hohe Vitamin E-Spiegel in den Spermien mit einer besseren Motilität und Morphologie assoziiert. Ein Zusammenhang zwischen der Konzentration im Seminalplasma und klassischen Spermiogrammparametern fand sich jedoch nur beim Vergleich gesunder Männer mit Patienten, die eine Oligozoospermie, Azoospermie bzw. Asthenozoospermie aufwiesen (Omu et al. 1999; Therond et al. 1996).

Wie bei Vitamin C sind infolge des höheren oxidativen Stresses bei Rauchern die $\alpha$ Tocopherol-Spiegel signifikant erniedrigt (Fraga et al. 1996).

Auch wenn eine einzelne randomisierte, doppelblind Placebo-kontrollierte Studie unter oraler Gabe von $300 \mathrm{mg}$ Vitamin E einen signifikanten Anstieg die Spermienmotilität bei gleichzeitig signifikanter Verringerung der Lipidperoxidation zeigen konnte (Suleiman et al. 1996), bleibt dieser Effekt kritisch zu hinterfragen. Die orale Einnahme von $600 \mathrm{mg}$ Vitamin E pro Tag führte in einer weiteren randomisierten, doppelblind Placebo-kontrollierten Studie zwar zur Konzentrationserhöhung im Serum, jedoch nicht im Seminalplasma (Kessopoulou et al. 1995).

Hinzu kommt, dass in anderen Studien meist eine Kombination aus Vitamin C und Vitamin E verabreicht wurde und es nicht möglich ist, die Wirkung dieser beiden Vitamine zu diskriminieren.

Am Rande sei erwähnt, dass die Zugabe des Antioxidanz Vitamin E zu Spermienseparationsmedien und Kryokonservierungsmedien aufgrund einer verminderten Lipidperoxidation die DNA-Integrität der Spermien (Hughes et al. 1998; Hughes et al. 1999) sowie im Tierexperiment auch Motilität und Vitalität verbessert (Jeong et al. 2009; Pena et al. 2003; Satorre et al. 2009).

\subsection{Coenzym $Q_{10}$}

Coenzym $\mathrm{Q}_{10}\left(\mathrm{CoQ}_{10}\right.$, syn. Ubichinon) ist Teil der mitochondrialen Atmungskette. Wie alle Zellen besitzen Spermien Mitochondrien für die Energiegewinnung. Sie befinden sich im Mittelstück und ermöglichen u.a. die aktive Beweglichkeit der männlichen Gameten (Fawcett 1975). Schädigende Einflüsse an dieser sensiblen Stelle kön- 
nen sehr leicht zum Motilitätsverlust durch Aktivierung der Apoptosesignalkaskade führen (Grunewald et al. 2006; Paasch et al. 2004). In diesem Zusammenhang entstand die Hypothese, dass $\mathrm{CoQ}_{10}$ neben den bekannten antioxidativen Eigenschaften durch Hemmung der Lipidperoxidation (Ernster u. Dallner 1995) eine Rolle bei der Spermienmotilität spielt.

$\mathrm{CoQ}_{10}$ findet man im Seminalplasma und in humanen Spermien (Alleva et al. 1997; Mancini et al. 1994). Dabei gibt es Unterschiede zwischen fertilen und infertilen Männern (Angelitti et al. 1995; Mancini et al. 1994). Studien zur Konzentration von $\mathrm{CoQ}_{10}$ in Spermien und im Seminalplasma bei Patienten mit Varicocele im Vergleich zu gesunden Kontrollen kamen zu konträren (Spiegel höher, gleich bzw. niedriger) Ergebnissen (Balercia et al. 2002; Mancini et al. 1994; Mancini et al. 2003; Mancini et al. 2005).

Im Hinblick auf die klassischen Spermienparameter fand sich eine signifikante Korrelation zwischen dem $\mathrm{CoQ}_{10}$ Spiegel im Ejakulat und der Anzahl der Spermatozoen bei gesunden Probanden und Patienten mit einer Varicocele (Angelitti et al. 1995; Mancini et al. 1994; Mancini et al. 2003). Bei Letzteren korrelierte der $\mathrm{CoQ}_{10}$-Gehalt in den Spermien zudem invers mit deren Motilität (Mancini et al. 2003; Mancini et al. 2005).

Bisher gibt es nur wenige Studien, die die Wirkung einer oralen Supplementierung mit $\mathrm{CoQ}_{10}$ bei infertilen Männern untersucht haben. Es wurde jedoch gezeigt, dass die $\mathrm{CoQ}_{10}$-Aufnahme zu einem signifikanten Anstieg des $\mathrm{CoQ}_{10}$-Gehaltes in den Spermien und im Seminalplasma führte (Balercia et al. 2004; Balercia et al. 2009) und die Motilität verbesserte (Balercia et al. 2004; Balercia et al. 2009). Möglicherweise hat $\mathrm{CoQ}_{10}$ einen positiven Effekt auf die Spermien von Männern mit Asthenozoospermie, weil gerade Männer mit sehr niedrigen Ausgangswerten bei der Motilität und $\mathrm{CoQ}_{10}{ }^{-}$ Spiegeln mit hoher Wahrscheinlichkeit auf die orale Therapie ansprechen (Balercia et al. 2009).

\subsection{Langkettige Fettsäuren}

In den terminal differenzierten Spermatozoen kommen zahlreiche Fettsäuren vor, u.a. Docosahexaensäure (DHA), Palmitinsäure, Linolensäure, Arachidonsäure, Linolsäure und Stearinsäure (Tavilani et al. 2006).

Phospholipid-gebundene DHA ist für die Fluidität der Spermienmembran von Bedeutung (Connor et al. 1998; Ollero et al. 200o). Besonders langkettige Fettsäuren (LCFA) sind wichtig für die Biegsamkeit und Flexibilität des Spermiumschwanzes und haben somit eine Bedeutung für die Spermienmotilität (Connor et al. 1998). Die DHA ist die Haupt-LCFA in humanen Spermatozoen (Lenzi et al. 200o; Tavilani et al. 2006; Zalata et al. 1998) und kommt in hoher Konzentration intrazellulär vor (Alvarez u. Storey 1995; Conquer et al. 1999; Zalata et al. 1998). DHA kann bis zu 14,3\% der Phospholipide im Spermium ausmachen (Lenzi et al. 2000; Zalata et al. 1998) und ist v.a. im Kopf lokalisiert (Zalata et al. 1998).

Bei Männern mit Asthenozoospermie waren die DHA-Spiegel in den Spermatozoen (Aksoy et al. 2006; Conquer et al. 1999; Zalata et al. 1998) und im Seminalplasma (Conquer et al. 1999) geringer als bei Männern mit Normozoospermie. Der DHA-Gehalt korrelierte signifikant mit der Spermienmotilität (Aksoy et al. 20o6; Conquer et al. 
1999; Tavilani et al. 2006; Zalata et al. 1998), Spermienkonzentration (Aksoy et al. 2006) und Spermienmorphologie (Aksoy et al. 2006; Tavilani et al. 2006; Zalata et al. 1998).

DHA nimmt zudem wahrscheinlich eine entscheidende Rolle bei der Spermiogenese ein. Während der Reifung der Spermien im Nebenhoden verändern sich Membranfluidität und die Phospholipid-Zusammensetzung der Membran (Hall et al. 1991). Bei immaturen Keimzellen sind die DHA-Gehalte in den Spermien höher als in den maturen Spermien (Ollero et al. 200o). Während der Spermienreifung wird DHA aus der Spermienmembran entfernt, die Anfälligkeit der Spermien für Lipidperoxidation sinkt (Ollero et al. 200o). Es ist bekannt, dass sich das Zytoplasma während der Spermienreifung im Spermienkopf verringert, wodurch auch die Membranlipide verloren gehen (Gergely et al. 1999; Huszar et al. 1997).

Möglicherweise ist die DHA-Synthese bei infertilen Männern vermindert. Durch Supplementierung von DHA kam es jedoch zu keinem Anstieg des DHA-Gehaltes in den Spermien von Männern mit Asthenozoospermie und auch die Motilität bzw. die Spermienzahl veränderten sich nicht (Conquer et al. 200o).

Nach der aktuellen Datenlage zeigt die orale Supplementierung langkettiger Fettsäuren keine Wirkung im Sinne einer therapeutischen Beeinflussbarkeit der klassischen Spermiogrammvariablen.

\section{Zusammenfassung}

Zum gegenwärtigen Zeitpunkt ist festzustellen, dass bei schwacher Datenlage eine Therapie mit Carnitin, Zink oder Coenzym $Q_{10}$ möglicherweise unterstützend in der Behandlung von Patienten mit einer Asthenozoospermie sein könnte. Bei Patienten mit einer hohen DNA-Fragmentationsrate kann die orale Gabe von Folsäure und Vitamin $\mathrm{C}$ versucht werden.

Bei den zitierten Studien ist zu berücksichtigen, dass die Mengen, die den Probanden verabreicht wurden, weit über dem von der Deutschen Gesellschaft für Ernährung empfohlenen Tagesbedarf lagen und somit über den physiologischen Bedarf hinausgingen. Dabei kann es auch zu unerwünschten Nebeneffekten kommen. Beispielsweise kann eine Überdosierung von Vitamin E die Thrombozytenaggregation hemmen und die Blutungsneigung steigern. Toxische Effekte von Selen zeigen sich in Neuropathien, Durchfall und Erbrechen.

Große klinisch kontrollierte randomisierte Studien wären notwendig, um weitere Effekte, aber auch die optimale Dosierung der einzelnen Nahrungsergänzungsmittel zu klären und damit Sicherheit und Wirkung zu gewährleisten.

\section{Literatur}

Akinloye 0, Arowojolu A0, Shittu OB, Adejuwon CA, Osotimehin B (2005) Selenium status of idiopathic infertile Nigerian males. Biol Trace Elem Res 104, 9-18

Aksoy Y, Aksoy H, Altinkaynak K, Aydin HR, Ozkan A (2006) Sperm fatty acid composition in subfertile men. Prostaglandins Leukot Essent Fatty Acids 75, 75-79

Alleva R, Scararmucci A, Mantero F, Bompadre S, Leoni L, Littarru GP (1997) The protective role of ubiquinol-10 against formation of lipid hydroperoxides in human seminal fluid. Mol Aspects Med 18 Suppl, S221-S228

Alvarez JG, Storey BT (1989) Role of glutathione peroxidase in protecting mammalian spermatozoa from loss of motility caused by spontaneous lipid peroxidation. Gamete Res 23, 77-90 
Alvarez IG, Storey BT (1995) Differential incorporation of fatty acids into and peroxidative loss of fatty acids from phospholipids of human spermatozoa. Mol Reprod Dev 42, 334-346

Angelitti AG, Colacicco L, Calla C, Arizzi M, Lippa S (1995) Coenzyme Q: potentially useful index of bioenergetic and oxidative status of spermatozoa. Clin Chem 41, 217-219

Balercia G, Arnaldi G, Fazioli F, Serresi M, Alleva R, Mancini A, Mosca F, Lamonica GR, Mantero F, Littarru GP (2002) Coenzyme Q10 levels in idiopathic and varicocele-associated asthenozoospermia. Andrologia 34, 107-111

Balercia G, Buldreghini E, Vignini A, Tiano L, Paggi F, Amoroso S, Ricciardo-Lamonica G, Boscaro M, Lenzi A, Littarru $G$ (2009) Coenzyme Q10 treatment in infertile men with idiopathic asthenozoospermia: a placebocontrolled, double-blind randomized trial. Fertil Steril 91, 1785-1792

Balercia G, Mosca F, Mantero F, Boscaro M, Mancini A, Ricciardo-Lamonica G, Littarru G (2004) Coenzyme Q(10) supplementation in infertile men with idiopathic asthenozoospermia: an open, uncontrolled pilot study. Fertil Steril 81, 93-98

Barney GH, Orgebin-Crist MC, Macapinalac MP (1968) Genesis of esophageal parakeratosis and histologic changes in the testes of the zinc-deficient rat and their reversal by zinc repletion. I Nutr 95, 526-534

Behne D, Gessner H, Wolters G, Brotherton I (1988) Selenium, rubidium and zinc in human semen and semen fractions. Int | Androl 11, 415-423

Bentivoglio G, Melica F, Cristoforoni P (1993) Folinic acid in the treatment of human male infertility. Fertil Steril $60,698-701$

Bleau G, Lemarbre J, Faucher G, Roberts KD, Chapdelaine A (1984). Semen selenium and human fertility. Fertil Steril 42, 890-894

Boxmeer |C, Smit M, Utomo E, Romijn |C, Eijkemans M|, Lindemans |, Laven |S, Macklon NS, Steegers EA, SteegersTheunissen RP (2009) Low folate in seminal plasma is associated with increased sperm DNA damage. Fertil Steril 92, 548-556

Brown DV, Senger PL, Stone SL, Froseth JA, Becker WC (1977) Glutatione peroxidase in bovine semen. I Reprod Fertil 50, 117-118

Cavallini G, Ferraretti AP, Gianaroli L, Biagiotti G, Vitali G (2004) Cinnoxicam and L-carnitine/acetyl-L-carnitine treatment for idiopathic and varicocele-associated oligoasthenospermia. I Androl 25, 761-770

Chia SE, Ong CN, Chua LH, Ho LM, Tay SK (2000) Comparison of zinc concentrations in blood and seminal plasma and the various sperm parameters between fertile and infertile men. I Androl 21, 53-57

Colagar AH, Marzony ET (2009) Ascorbic Acid in human seminal plasma: determination and its relationship to sperm quality. I Clin Biochem Nutr 45, 144-149

Colagar AH, Marzony Et, Chaichi MJ (2009) Zinc levels in seminal plasma are associated with sperm quality in fertile and infertile men. Nutr Res 29, 82-88

Connor WE, Lin DS, Wolf DP, Alexander M (1998) Uneven distribution of desmosterol and docosahexaenoic acid in the heads and tails of monkey sperm. I Lipid Res 39, 1404-1411

Conquer JA, Martin JB, Tummon I, Watson L, Tekpetey F (1999) Fatty acid analysis of blood serum, seminal plasma, and spermatozoa of normozoospermic vs. asthenozoospermic males. Lipids 34, 793-799

Conquer IA, Martin JB, Tummon I, Watson L, Tekpetey F (2000) Effect of DHA supplementation on DHA status and sperm motility in asthenozoospermic males. Lipids 35, 149-154

Costa M, Canale D, Filicori M, D'lddio S, Lenzi A (1994) L-carnitine in idiopathic asthenozoospermia: a multicenter study. Italian Study Group on Carnitine and Male Infertility. Andrologia 26, 155-159

Dawson EB, Harris WA, Rankin WE, Charpentier LA, McGanity WJ (1987) Effect of ascorbic acid on male fertility. Ann N Y Acad Sci 498, 312-323

De Bree A, van Dusseldorp M, Brouwer IA, van het Hof KH, Steegers-Theunissen RP (1997) Folate intake in Europe: recommended, actual and desired intake. Eur / Clin Nutr 51, 643-660

Ernster L, Dallner G (1995) Biochemical, physiological and medical aspects of ubiquinone function. Biochim Biophys Acta 1271, 195-204

Fawcett DW (1975) The mammalian spermatozoon. Dev Biol 44, 394-436

Flohe L, Gunzler WA, Schock HH (1973) Glutathione peroxidase: a selenoenzyme. FEBS Lett 32, 132-134

Foresta C, Flohe L, Garolla A, Roveri A, Ursini F, Maiorino M (2002) Male fertility is linked to the selenoprotein phospholipid hydroperoxide glutathione peroxidase. Biol Reprod 67, 967-971 
Fraga CG, Motchnik PA, Shigenaga MK, Helbock HJ, Jacob RA, Ames BN (1991) Ascorbic acid protects against endogenous oxidative DNA damage in human sperm. Proc Natl Acad Sci U.S.A. 88, 11003-11006

Fraga CG, Motchnik PA, Wyrobek A), Rempel DM, Ames BN (1996) Smoking and low antioxidant levels increase oxidative damage to sperm DNA. Mutat Res 351, 199-203

Fuse H, Kazama T, Ohta S, Fujiuchi Y (1999) Relationship between zinc concentrations in seminal plasma and various sperm parameters. Int Urol Nephrol 31, 401-408

Gavella M, Lipovac V (1998) In vitro effect of zinc on oxidative changes in human semen. Andrologia 30, 317-323

Gergely A, Kovanci E, Senturk L, Cosmi E, Vigue L, Huszar G (1999) Morphometric assessment of mature and diminished-maturity human spermatozoa: sperm regions that reflect differences in maturity. Hum Reprod $14,2007-2014$

Grunewald S, Paasch U, Said TM, Rasch M, Agarwal A, Glander HJ (2006) Magnetic-activated Cell Sorting before Cryopreservation Preserves Mitochondrial Integrity in Human Spermatozoa. Cell Tissue Bank 7, 99-104

Hall IC, Hadley I, Doman T (1991) Correlation between changes in rat sperm membrane lipids, protein, and the membrane physical state during epididymal maturation. I Androl 12, 76-87

Hinton BT, Brooks DE, Dott HM, Setchell BP (1981) Effects of carnitine and some related compounds on the motility of rat spermatozoa from the caput epididymidis. I Reprod Fertil 61, 59-64

Hughes CM, Lewis SEM, McKelvey-Martin VI, Thompson W (1998) The effects of antioxidant supplementation during Percoll preparation on human sperm DNA integrity. Hum Reprod 13, 1240-1247

Hughes CM, McKelvey-Martin VI, Lewis SE (1999) Human sperm DNA integrity assessed by the Comet and ELI assays. Mutagenesis

Huszar G, Sbracia M, Vigue L, Miller DI, Shur BD (1997) Sperm plasma membrane remodeling during spermiogenetic maturation in men: relationship among plasma membrane beta 1,4-galactosyltransferase, cytoplasmic creatine phosphokinase, and creatine phosphokinase isoform ratios. Biol Reprod 56, 1020-1024

Iwanier K, Zachara BA (1995) Selenium supplementation enhances the element concentration in blood and seminal fluid but does not change the spermatozoal quality characteristics in subfertile men. | Androl 16, 441-447

Jeong YJ, Kim MK, Song HI, Kang El, Ock SA, Kumar BM, Balasubramanian S, Rho G) (2009) Effect of alpha-tocopherol supplementation during boar semen cryopreservation on sperm characteristics and expression of apoptosis related genes. Cryobiology 58, 181-189

Jeulin C, Soufir JC, Marson J, Paquignon M, Dacheux JL (1987) The distribution of carnitine and acetylcarnitine in the epididymis and epididymal spermatozoa of the boar. I Reprod Fertil 79, 523-529

Kao SH, Chao HT, Chen HW, Hwang TI, Liao TL, Wei YH (2008) Increase of oxidative stress in human sperm with lower motility. Fertil Steril 89, 1183-1190

Kessopoulou E, Powers HJ, Sharma KK, Pearson MJ, Russell JM, Cooke ID, Barratt CL (1995) A double-blind randomized placebo cross-over controlled trial using the antioxidant vitamin $\mathrm{E}$ to treat reactive oxygen species associated male infertility. Fertil Steril 64, 825-831

Khademi A, Alleyassin A, Safdarian L, Hamed EA, Rabiee E, Haghaninezhad H (2005) The effects of L-carnitine on sperm parameters in smoker and non-smoker patients with idiopathic sperm abnormalities. I Assist Reprod Genet 22, 395-399

Kohengkul S, Tanphaichitr V, Muangmun V, Tanphaichitr N (1977) Levels of L-carnitine and L-O-acetylcarnitine in normal and infertile human semen: a lower level of L-O-acetycarnitine in infertile semen. Fertil Steril 28, $1333-1336$

Kvist U, Kjellberg S, Bjorndahl L, Soufir IC, Arver S (1990) Seminal fluid from men with agenesis of the Wolffian ducts: zinc-binding properties and effects on sperm chromatin stability. Int I Androl 13, 245-252

Kynaston HG, Lewis-lones DI, Lynch RV, Desmond AD (1988) Changes in seminal quality following oral zinc therapy. Andrologia 20, 21-22

Landau B, Singer R, Klein T, Segenreich E (1978) Folic acid levels in blood and seminal plasma of normo- and oligospermic patients prior and following folic acid treatment. Experientia 34, 1301-1302

Lenzi A, Gandini L, Maresca V, Rago R, Sgro P, Dondero F, Picardo M (2000) Fatty acid composition of spermatozoa and immature germ cells. Mol Hum Reprod 6, 226-231

Lenzi A, Lombardo F, Sgro P, Salacone P, Caponecchia L, Dondero F, Gandini L (2003) Use of carnitine therapy in selected cases of male factor infertility: a double-blind crossover trial. Fertil Steril 79, 292-300 
Lenzi A, Sgro P, Salacone P, Paoli D, Gilio B, Lombardo F, Santulli M, Agarwal A, Gandini L (2004) A placebocontrolled double-blind randomized trial of the use of combined l-carnitine and l-acetyl-carnitine treatment in men with asthenozoospermia. Fertil Steril 81, 1578-1584

Lewis SE, Sterling ES, Young IS, Thompson W (1997) Comparison of individual antioxidants of sperm and seminal plasma in fertile and infertile men. Fertil Steril 67, 142-147

Liu DY, Sie BS, Liu ML, Agresta F, Baker HW (2009) Relationship between seminal plasma zinc concentration and spermatozoa-zona pellucida binding and the ZP-induced acrosome reaction in subfertile men. Asian I Androl 11, 499-507

Maiorino M, Coassin M, Roveri A, Ursini F (1989) Microsomal lipid peroxidation: effect of vitamin E and its functional interaction with phospholipid hydroperoxide glutathione peroxidase. Lipids 24, 721-726

Mancini A, De ML, Oradei A, Hallgass ME, Conte G, Pozza D, Littarru GP (1994) Coenzyme Q10 concentrations in normal and pathological human seminal fluid. I Androl 15, 591-594

Mancini A, Milardi D, Conte G, Bianchi A, Balercia G, De ML, Littarru GP (2003) Coenzyme Q10: another biochemical alteration linked to infertility in varicocele patients? Metabolism 52, 402-406

Mancini A, Milardi D, Conte G, Festa R, De ML, Littarru GP (2005) Seminal antioxidants in humans: preoperative and postoperative evaluation of coenzyme Q10 in varicocele patients. Horm Metab Res 37, 428-432

Menkveld R, Stander FS, Kotze TJ, Kruger TF, van Zyl JA (1990) The evaluation of morphological characteristics of human spermatozoa according to stricter criteria. Hum Reprod 5, 586-592

Mostafa T, Tawadrous G, Roaia MM, Amer MK, Kader RA, Aziz A (2006) Effect of smoking on seminal plasma ascorbic acid in infertile and fertile males. Andrologia 38, 221-224

Noack-Fuller G, De BC, Seibert H (1993) Cadmium, lead, selenium, and zinc in semen of occupationally unexposed men. Andrologia 25, 7-12

Oldereid NB, Thomassen Y, Purvis K (1998) Selenium in human male reproductive organs. Hum Reprod 13, 21722176

Ollero M, Powers RD, Alvarez IG (2000) Variation of docosahexaenoic acid content in subsets of human spermatozoa at different stages of maturation: implications for sperm lipoperoxidative damage. Mol Reprod Dev 55, 326-334

Omu AE, Al-Azemi MK, Kehinde EO, Anim JT, Oriowo MA, Mathew TC (2008) Indications of the mechanisms involved in improved sperm parameters by zinc therapy. Med Princ Pract 17, 108-116

Omu AE, Dashti H, Al-Othman S (1998) Treatment of asthenozoospermia with zinc sulphate: andrological, immunological and obstetric outcome. Eur | Obstet Gynecol Reprod Biol 79, 179-184

Omu AE, Fatinikun T, Mannazhath N, Abraham S (1999) Significance of simultaneous determination of serum and seminal plasma alpha-tocopherol and retinol in infertile men by high-performance liquid chromatography. Andrologia 31, 347-354

Paasch U, Agarwal A, Gupta AK, Sharma RK, Grunewald S, Thomas Al Jr., Glander HJ (2004) Apoptosis signal transduction and the maturity status of human spermatozoa. Ann NY Acad Sci 1010, 486-489

Pena FI, Johannisson A, Wallgren M, Rodriguez MH (2003) Antioxidant supplementation in vitro improves boar sperm motility and mitochondrial membrane potential after cryopreservation of different fractions of the ejaculate. Anim Reprod Sci 78, 85-98

Piomboni P, Gambera L, Serafini F, Campanella G, Morgante G, De Leo V (2008) Sperm quality improvement after natural anti-oxidant treatment of asthenoteratospermic men with leukocytospermia. Asian I Androl 10, 201-206

Rao B, Soufir JC, Martin M, David G (1989) Lipid peroxidation in human spermatozoa as related to midpiece abnormalities and motility. Gamete Res 24, 127-134

Rotruck JT, Pope AL, Ganther HE, Swanson AB, Hafeman DG, Hoekstra WG (1973) Selenium: biochemical role as a component of glutathione peroxidase. Science 179, 588-590

Roy AC, Karunanithy R, Ratnam SS (1990) Lack of correlation of selenium level in human semen with sperm count/motility. Arch Androl 25, 59-62

Saaranen M, Suistomaa U, Kantola M, Saarikoski S, Vanha-Perttula T (1987) Lead, magnesium, selenium and zinc in human seminal fluid: comparison with semen parameters and fertility. Hum Reprod 2, 475-479

Saaranen M, Suistomaa U, Vanha-Perttula T (1989) Semen selenium content and sperm mitochondrial volume in human and some animal species. Hum Reprod 4, 304-308 
Sandstead HH, Prasad AS, Schulert AR, Farid Z, Miale A Jr., Bassilly S, Darby WJ (1967) Human zinc deficiency, endocrine manifestations and response to treatment. Am / Clin Nutr 20, 422-442

Satorre MM, Breininger E, Beconi MT, Beorlegui NB (2009) Protein tyrosine phosphorylation under capacitating conditions in porcine fresh spermatozoa and sperm cryopreserved with and without alpha tocopherol. Andrologia 41, 184-192

Scott R, MacPherson A, Yates RW, Hussain B, Dixon I (1998) The effect of oral selenium supplementation on human sperm motility. Br J Urol 82, 76-80

Shaarawy M, Mahmoud KZ (1982) Endocrine profile and semen characteristics in male smokers. Fertil Steril 38, $255-257$

Sheikh N, Goodarzi MT, Bab Al-Havaejee H, Safari MR, Amiri I, Najafi R, Hadeie I (2007) L-Carnitine Level in Seminal Plasma of Fertile and Infertile Men. I Res Health Sci 7, 43-48

Siliprandi N, Sartorelli L, Ciman M, Di LF (1989) Carnitine: metabolism and clinical chemistry. Clin Chim Acta 183, 3-11

Song G], Norkus EP, Lewis V (2006) Relationship between seminal ascorbic acid and sperm DNA integrity in infertile men. Int I Androl 29, 569-575

Suleiman SA, Ali ME, Zaki ZM, el-Malik EM, Nasr MA (1996) Lipid peroxidation and human sperm motility: protective role of vitamin E. I Androl 17, 530-537

Tavilani H, Doosti M, Abdi K, Vaisiraygani A, Joshaghani HR (2006) Decreased polyunsaturated and increased saturated fatty acid concentration in spermatozoa from asthenozoospermic males as compared with ormozoospermic males. Andrologia 38, 173-178

Therond P, Auger I, Legrand A, Jouannet P (1996) alpha-Tocopherol in human spermatozoa and seminal plasma: relationships with motility, antioxidant enzymes and leukocytes. Mol Hum Reprod 2, 739-744

Thiele ||, Friesleben HI, Fuchs |, Ochsendorf FR (1995) Ascorbic acid and urate in human seminal plasma: determination and interrelationships with chemiluminescence in washed semen. Hum Reprod 10, 110-115

Thomas IP, Maiorino M, Ursini F, Girotti AW (1990) Protective action of phospholipid hydroperoxide glutathione peroxidase against membrane-damaging lipid peroxidation. In situ reduction of phospholipid and cholesterol hydroperoxides. I Biol Chem 265, 454-461

Tikkiwal M, Ajmera RL, Mathur NK (1987) Effect of zinc administration on seminal zinc and fertility of oligospermic males. Indian | Physiol Pharmacol 31, 30-34

Vicari E, Calogero AE (2001) Effects of treatment with carnitines in infertile patients with prostato-vesiculo-epididymitis. Hum Reprod 16, 2338-2342

Wallock LM, Tamura T, Mayr CA, Johnston KE, Ames BN, Jacob RA (2001) Low seminal plasma folate concentrations are associated with low sperm density and count in male smokers and nonsmokers. Fertil Steril 75, 252-259

Wong WY, Flik G, Groenen PM, Swinkels DW, Thomas DM, Copius-Peereboom JH, Merkus HM, Steegers-Theunissen RP (2001) The impact of calcium, magnesium, zinc, and copper in blood and seminal plasma on semen parameters in men. Reprod Toxicol 15, 131-136

Wong WY, Merkus HM, Thomas CM, Menkveld R, Zielhuis GA, Steegers-Theunissen RP (2002) Effects of folic acid and zinc sulfate on male factor subfertility: a double-blind, randomized, placebo-controlled trial. Fertil Steril 77, 491-498

Xu B, Chia SE, Ong CN (1994) Concentrations of cadmium, lead, selenium, and zinc in human blood and seminal plasma. Biol Trace Elem Res 40, 49-57

Xu B, Chia SE, Tsakok M, Ong CN (1993) Trace elements in blood and seminal plasma and their relationship to sperm quality. Reprod Toxicol 7, 613-618

Xu DX, Shen HM, Zhu QX, Chua L, Wang QN, Chia SE, Ong CN (2003) The associations among semen quality, oxidative DNA damage in human spermatozoa and concentrations of cadmium, lead and selenium in seminal plasma. Mutat Res 534, 155-163

Xuan W, Lamhonwah AM, Librach C, Jarvi K, Tein I (2003) Characterization of organic cation/carnitine transporter family in human sperm. Biochem Biophys Res Commun 306, 121-128

Zalata AA, Christophe AB, Depuydt CE, Schoonjans F, Comhaire FH (1998) The fatty acid composition of phospholipids of spermatozoa from infertile patients. Mol Hum Reprod 4, 111-118

Zopfgen A, Priem F, Sudhoff F, Jung K, Lenk S, Loening SA, Sinha P (2000) Relationship between semen quality and the seminal plasma components carnitine, alpha-glucosidase, fructose, citrate and granulocyte elastase in infertile men compared with a normal population. Hum Reprod 15, 840-845 